\title{
Consumo habitual de café y riesgo de eventos cerebrovasculares
}

\author{
Regular coffee consumption and stroke risk \\ Nora Badoui, Luz H. Alba*, Juan S. Castillo, Maylin Peñaloza, Vanesa Gutiérrez y Adriana Ibarra \\ Departamento de Medicina Preventiva, Facultad de Medicina, Universidad Javeriana, Bogotá, Colombia
}

\section{Resumen}

Introducción: La enfermedad cerebrovascular es causa frecuente de morbimortalidad y, en ese sentido, el consumo de café tiene un impacto cardiovascular, por lo cual es importante evaluar la evidencia respecto a la asociación entre su consumo y la enfermedad cerebrovascular. Objetivo: Evaluar la asociación entre consumo de café y riesgo de morbimortalidad por enfermedad cerebrovascular. Método: Se realizó una búsqueda en las bases Medline, EMBASE, LILACS y Cochrane (enero de 1966 a junio de 2018) y se seleccionaron revisiones sistemáticas y metaanálisis evaluados de forma estandarizada y pareada. Se seleccionaron seis publicaciones. Resultados: Se encontró que el consumo de café en rango moderado (hasta cuatro tazas) se asocia a una reducción del riesgo de enfermedad cerebrovascular (riesgo relativo [RR] $=0.89$, intervalo de confianza del 95\% [IC95\%]: 0.81-0.97, y RR: 0.83, IC95\%: 0.75-0.91). Esta protección se mantiene en el subgrupo de mujeres, con reducciones del 13\% (IC95\%: 0.78-0.97) para una taza, del 16\% (IC95\%: 0.74-0.95) para dos tazas y 19\% (RR:0.81; IC95\%: 0.70-0.93) (IC95\%: 0.70-0.93) para cuatro o más tazas. Los hallazgos también son significativos para el subtipo isquémico ( $R R=0.80$; IC95\%: 0.71-0.90). Conclusiones: El consumo de café reduce el riesgo de eventos cerebrovasculares entre un $11 \%$ y un $17 \%$, y esto se mantiene en el subgrupo de mujeres y en el subtipo isquémico.

Palabras clave: Café. Accidente cerebrovascular. Mortalidad. Revisión.

\section{Abstract}

Introduction: Cerebrovascular disease is a frequent cause of morbidity and mortality and, in this sense, coffee consumption has a cardiovascular impact, which is why it is important to evaluate the evidence regarding the association between its consumption and cerebrovascular disease. Objective: To evaluate the association between coffee consumption and risk of morbidity and mortality due to cerebrovascular disease. Method: A search was carried out in the Medline, EMBASE, LILACS and Cochrane databases (January 1966 to June 2018), selecting systematic reviews and meta-analyzes evaluated in a standardized and paired way. Six publications were selected. Results: it was found that the consumption of coffee in a moderate range (up to 4 cups) is associated with a reduction in the risk of cerebrovascular disease (relative risk [RR] $=0.89,95 \%$ confidence interval [95\% Cl]: 0.81- 0.97, and $R R=0.83,95 \% \mathrm{Cl}: 0.75-0.91)$. This protection is maintained in the subgroup of women, with reductions of $13 \%$ (95\% Cl: 0.78 $0.97)$ for a cup, $16 \%$ (95\% Cl: 0.74-0.95) for two cups, and $R R=0.81$ (95\% Cl: 0.70-0.93) for four or more cups. The findings are also significant for the ischemic subtype $(R R=0.80 ; 95 \% \mathrm{Cl}$ : 0.71-0.90). Conclusions: Coffee consumption reduces the risk of cerebrovascular events between $11 \%$ and $17 \%$, and this is maintained in the subgroup of women and in the ischemic subtype.

Key words: Coffee. Stroke. Mortality. Revision.

\section{Correspondencia:}

*Luz H. Alba

E-mail: lalba@javeriana.edu.co
Disponible en internet: 18-10-2021 Rev Colomb Cardiol. 2021;28(4):389-396 www.rccardiologia.com 0120-5633 / C 2020 Sociedad Colombiana de Cardiología y Cirugía Cardiovascular. Publicado por Permanyer. Este es un artículo open access bajo la licencia CC BY-NC-ND (http://creativecommons.org/licenses/by-nc-nd/4.0/). 


\section{Introducción}

La enfermedad cerebrovascular se define por la presencia de déficit neurológico, focal o global, de etiología vascular, y adquiere importancia por el impacto que tiene en términos de discapacidad y muerte ${ }^{1}$. Es la segunda causa de muerte en todo el mundo, y además se reporta un incremento en su incidencia del 100\% para los países de ingresos medios y bajos. Según el Observatorio Nacional de Salud, para Colombia se estima una prevalencia de enfermedad cerebrovascular entre 1.4 y 19.9 casos por 100,000 habitantes, con una alta variabilidad según la fuente consultada, lo cual, de acuerdo con los datos del Departamento Administrativo Nacional de Estadística, corresponde a la segunda causa de muerte en el país ${ }^{2,3}$.

Los principales factores de riesgo identificados en el desarrollo de esta enfermedad son la hipertensión arterial, la enfermedad cardiovascular isquémica y arrítmica, la diabetes mellitus y el tabaquismo, entre otros $^{1-3}$. Se sabe que la intervención sobre estos factores de riesgo conduce a una reducción de la aparición de la enfermedad; sin embargo, es interesante identificar otras intervenciones complementarias que reduzcan el riesgo de enfermedad cerebrovascular.

En vista de que el café es una de las bebidas de mayor consumo en el mundo $0^{4,5}$, su relación con desenlaces tanto positivos como negativos puede tener consecuencias en la salud pública.

Colombia es uno de los principales países productores de café y cuenta con un consumo importante. En el estudio del programa Toma Café de 2015 se reportaron promedios de consumo de 3-4 tazas al día por persona. Adicionalmente, alrededor de este consumo hay factores sociales y culturales significativos, así como mitos sobre el impacto de su consumo y los desenlaces en salud, por lo cual es común que a los pacientes con enfermedad cardiovascular o hipertensiva se les proscriba esta bebida $^{6-8}$. No obstante, el café, el té y el chocolate han sido estudiados por su potencial efecto benéfico cardiovascular, y diversas investigaciones que analizan la relación entre consumo de café y salud han encontrado efectos positivos en cuanto a mortalidad, algunas neoplasias y desenlaces cardiovasculares, entre otros ${ }^{9-11}$.

Una de las teorías que explican este beneficio son los polifenoles, compuestos presentes en estos alimentos, que son nutrientes que se pueden obtener de diversas fuentes y tienen un efecto vascular demostrado, puesto que incrementan la respuesta vasodilatadora mediada por acetilcolina y óxido nítrico. También hay evidencia de su efecto respecto al perfil lipídico, con reducciones en el colesterol total y el colesterol unido a lipoproteínas de baja densidad, y el incremento en antioxidantes, entre otros ${ }^{12-14}$.

En el caso del café, el principal polifenol es el ácido clorogénico, que ejerce efectos antioxidantes, y si bien algunos estudios han encontrado una correlación entre el consumo de café y el incremento de la presión arterial (mediado por la cafeína), este parece ser un fenómeno transitorio y, en el largo plazo, el efecto neto parece ser benéfico en virtud del efecto vasodilatador del ácido clorogénico ${ }^{13,15,16}$.

Con base en lo anterior, está claro por qué resulta importante hacer una revisión de la evidencia disponible y evaluar cómo se relaciona el consumo habitual de café con la salud, específicamente con el riesgo de eventos cerebrovasculares.

\section{Presentación del caso}

Mujer de 65 años, asintomática, que acude para cumplir con el control periódico de su hipertensión arterial, la cual tiene 8 años de evolución. Recibe tratamiento con losartán (50 mg/12 h). No tiene otras enfermedades diagnosticadas ni toma otros medicamentos. Su exploración física no revela ninguna alteración significativa y sus signos vitales están estables. Manifiesta estar preocupada porque su padre tuvo un «derrame» cerebral y escuchó que esto podría tener alguna relación con la alta ingesta de café; él consumía entre 4 y 6 tazas diarias de café y ella suele tomar 2 o 3 tazas.

Refiere que disfruta enormemente de tomar café y que no desearía dejarlo, pero le preocupa que esto pueda incrementar su riesgo de eventos cerebrovasculares. Su preocupación la hizo cambiar a un café liofilizado y descafeinado, esperando que esto tenga menor riesgo respecto al café filtrado y los expresos que solía tomar.

Usted se pregunta si el consumo de café tiene algún efecto sobre la aparición de eventos cerebrovasculares y si esto varía según el tipo de café o de preparación.

\section{Método}

Se realizó una búsqueda de la literatura en las bases de datos Medline a través de PubMed, EMBASE, LILACS y la Organización Cochrane, entre enero de 1966 y diciembre de 2017, usando los términos MESH (Medical Subject Headings) "coffee, stroke, cerebrovasc*, mortality". Se limitó a los idiomas inglés, español, francés y portugués. Adicionalmente, se realizó una búsqueda manual en las referencias de los estudios seleccionados. 
Se incluyeron artículos con población tanto masculina como femenina, mayor de 18 años. La intervención evaluada en los estudios fue el consumo habitual 0 crónico de café frente al no consumo o el consumo mínimo (una dosis inferior a la dosis a comparar). Se excluyeron mujeres embarazadas y en periodo de lactancia, personas con trastornos mentales y dependencia de sustancias psicoactivas, personas con diagnóstico de cáncer de cualquier localización, y pacientes usuarios habituales de medicamentos con cafeína o de bebidas diferentes del café que contuvieran esta sustancia. No se limitó por ninguna raza ni región ${ }^{17}$.

Se hizo una selección inicial por títulos y resúmenes, y tras identificar aquellos relacionados con el tema se obtuvieron las publicaciones completas. Se evaluó y calificó cada publicación de forma pareada usando el instrumento AMSTAR (Assessing Methodologycal Quality for Systematic Reviews). Se incluyeron aquellos estudios con una calificación de moderada a alta calidad (puntuación AMSTAR $\geq 7 / 11$ ). El equipo evaluador estuvo integrado por profesionales con formación en epidemiología, estadística, salud pública y medicina familiar.

En esta publicación se resumen los resultados de aquellos artículos cuyo desenlace primario fue la aparición de eventos cerebrovasculares y mortalidad, o ambos, por enfermedad cerebrovascular, y que cumplieron con el criterio de calidad. Acorde con las descripciones de las revisiones incluidas y el comportamiento de los desenlaces, y en aras de facilitar la interpretación de los resultados por parte de los lectores, se agrupan las categorías de consumo de la siguiente forma: consumo ligero de café como aquel de 3 tazas o menos al día, consumo moderado como aquel entre 3 y 4 tazas al día, y consumo pesado como aquel de más de 4 tazas.

La búsqueda se actualizó en junio de 2018 con el fin de identificar nuevas publicaciones, pero no se encontraron otras referencias que cumplieran los criterios de inclusión para el tópico de consumo de café y eventos cerebrovasculares.

Para graduar la fortaleza de la evidencia final se utilizó el sistema GRADE (Grading of Recommendations, Assesment, Development and Evaluation), utilizando el software GRADEpro ${ }^{18}$.

Las figuras se diseñaron con GraphPad® versión 8.0.1 (Trial) (GraphPad Software, Inc., La Jolla, CA, USA $)^{19}$.

\section{Resultados}

La búsqueda inicial arrojó 177 artículos, de los cuales 72 correspondieron a revisiones sistemáticas relacionadas con el tema del café y la salud cardiovascular. De estos, se seleccionaron seis metaanálisis (cuyas publicaciones primarias fueron cohortes) que tenían por desenlace la aparición de enfermedad cerebrovascular o la mortalidad por esta causa (Tabla 1).

\section{Eventos cerebrovasculares}

Dos de los metaanálisis incluidos reportan riesgo de enfermedad cerebrovascular cuando se compara cualquier categoría de consumo con el no consumo. En ambos, la reducción del riesgo de enfermedad cerebrovascular es significativa, del $11 \%$ en el estudio de Zhang, et al. ${ }^{20}$ (que abarca el mayor número de regiones) y del $17 \%$ en el de Kim, et al. ${ }^{21}$ (Tabla 2).

En los análisis por subgrupos, la protección es diferente según las distintas categorías de consumo. Para un consumo ligero (considerado como aquel inferior a 3 tazas) se reportan reducciones del riesgo de enfermedad cerebrovascular significativas, con riesgo relativo $(\mathrm{RR})=$ 0.88 (intervalo de confianza del 95\% [IC95\%]: 0.86-0.90) y $R R=0.90$ (IC95\%: 0.85-0.96) $)^{20,22}$. La categoría de consumo de 3 a 4 tazas se asocia con reducciones entre el $5 \%$ y el $12 \%$, aunque no es una reducción significativa en todos los estudios; Zhang, et al. ${ }^{20}$ reportan RR $=0.86$ (IC95\%: 0.76-0.97), en tanto que para Larsson y Orsini ${ }^{22}$ la tendencia de reducción es marcada, pero sin significancia estadística (RR = 0.88; IC95\%: 0.77 a 1.01).

En tres de los metaanálisis revisados se incluyen categorías de consumo de 4 o más tazas, con las que hay evidencia de reducción del riesgo de enfermedad cerebrovascular significativa para dos de los estudios (RR = 0.83, IC95\%: 0.76-0.91, y RR = 0.83, IC95\%: 0.79$0.89)^{20,21}$, evidenciando una tendencia a la reducción del riesgo en el estudio de Larsson y Orsini ${ }^{22}(\mathrm{RR}=0.87$; IC95\%: 0.75-1.02). Este último estudio es el único que incluyó una categoría de consumo de 7 o más tazas, valor con el cual se mantiene la tendencia, pero no se asocia a reducciones significativas; adicionalmente, tras excluir aquellos estudios que incluyen pacientes con antecedente de infarto agudo de miocardio (dos publicaciones) y diabetes mellitus (una publicación), no se encuentran cambios significativos en los $\mathrm{RR}$ reportados (Tabla 2).

Por su parte, en el metaanálisis de Mesas, et al. ${ }^{23}$ tuvieron en consideración la asociación de hipertensión y consumo de café con la aparición de eventos cerebrovasculares, y encontraron resultados inconsistentes; de los estudios incluidos, uno reportó incremento del riesgo de eventos tromboembólicos con consumos de alrededor de 4 tazas al día, otro no encontró asociación, y un tercero halló un riesgo menor 
Rev Colomb Cardiol. 2021;28(4)

Tabla 1. Características de las revisiones sistemáticas y de los metaanálisis incluidos

\begin{tabular}{|c|c|c|c|c|c|c|c|c|}
\hline Estudio & $\begin{array}{l}\text { Países de } \\
\text { las } \\
\text { cohortes } \\
\text { incluidas }\end{array}$ & $\begin{array}{l}\text { Número de } \\
\text { participantes }\end{array}$ & $\begin{array}{l}\text { Población } \\
\text { incluida }\end{array}$ & Intervención & Comparador & $\begin{array}{l}\text { Desenlaces } \\
\text { evaluados }\end{array}$ & $\begin{array}{l}\text { Número } \\
\text { de } \\
\text { estudios } \\
\text { primarios } \\
\text { incluidos }\end{array}$ & $\begin{array}{l}\text { Calificación } \\
\text { de la } \\
\text { revisión } \\
\text { según } \\
\text { AMSTAR }\end{array}$ \\
\hline $\begin{array}{l}\text { Grosso, } \\
\text { et al., } \\
2016\end{array}$ & $\begin{array}{l}\text { Estados } \\
\text { Unidos, } \\
\text { Holanda, } \\
\text { Noruega, } \\
\text { Suecia, } \\
\text { Escocia, } \\
\text { Reino } \\
\text { Unido, } \\
\text { Finlandia, } \\
\text { Croacia, } \\
\text { República } \\
\text { Checa, } \\
\text { Rusia, } \\
\text { Polonia, } \\
\text { Japón, } \\
\text { Singapur }\end{array}$ & $1,610,543$ & $\begin{array}{l}\text { Población } \\
\text { adulta sana }\end{array}$ & $\begin{array}{l}\text { Consumo } \\
\text { habitual de } \\
\text { café en } \\
\text { diferentes } \\
\text { dosis: de } 1 \\
\text { hasta } 7 \\
\text { tazas al día }\end{array}$ & $\begin{array}{l}\text { No } \\
\text { consumo } \\
\text { de café }\end{array}$ & $\begin{array}{l}\text { Riesgo de } \\
\text { muerte por } \\
\text { ECV }\end{array}$ & 9 & $8 / 11$ \\
\hline $\begin{array}{l}\text { Malerba, } \\
\text { et al., } \\
2013\end{array}$ & $\begin{array}{l}\text { Estados } \\
\text { Unidos, } \\
\text { Holanda, } \\
\text { Suecia, } \\
\text { Escocia, } \\
\text { Finlandia, } \\
\text { Japón, } \\
\text { Croacia }\end{array}$ & $\begin{array}{l}\text { No } \\
\text { especifica }\end{array}$ & $\begin{array}{l}\text { Población } \\
\text { adulta sana }\end{array}$ & $\begin{array}{l}\text { Consumo } \\
\text { habitual de } \\
\text { café en } \\
\text { diferentes } \\
\text { dosis: menor } \\
\text { o mayor de } 3 \\
\text { tazas al día }\end{array}$ & $\begin{array}{l}\text { No } \\
\text { consumo } \\
\text { de café o la } \\
\text { categoría } \\
\text { más baja } \\
\text { de consumo } \\
\text { como } \\
\text { referencia }\end{array}$ & $\begin{array}{l}\text { Riesgo de } \\
\text { muerte por } \\
\text { ECV }\end{array}$ & 6 & $8 / 11$ \\
\hline $\begin{array}{l}\text { Larsson } \\
\text { y Orsini, } \\
2011\end{array}$ & $\begin{array}{l}\text { Europa, } \\
\text { Estados } \\
\text { Unidos, } \\
\text { Japón }\end{array}$ & 479,689 & $\begin{array}{l}\text { Población } \\
\text { general (8), } \\
\text { antecedente } \\
\text { de IAM } \\
\text { reciente (2), } \\
\text { antecedente } \\
\text { de DM2 (1) }\end{array}$ & $\begin{array}{l}\text { Consumo } \\
\text { habitual de } \\
\text { café en } \\
\text { diferentes } \\
\text { categorías: } \\
\text { de } 1 \text { a } 7 \text { o } \\
\text { más tazas al } \\
\text { día }\end{array}$ & $\begin{array}{l}\text { No } \\
\text { consumo } \\
\text { de café }\end{array}$ & Riesgo de ECV & 11 & $7 / 11$ \\
\hline $\begin{array}{l}\text { Kim, } \\
\text { et al., } \\
2012\end{array}$ & $\begin{array}{l}\text { Estados } \\
\text { Unidos, } \\
\text { Europa }\end{array}$ & 206,437 & $\begin{array}{l}\text { Población } \\
\text { adulta sana }\end{array}$ & $\begin{array}{l}\text { Consumo } \\
\text { habitual de } \\
\text { café en } \\
\text { diferentes } \\
\text { dosis: menor } \\
\text { o mayor de } 3 \\
\text { tazas al día }\end{array}$ & $\begin{array}{l}\text { No } \\
\text { consumo } \\
\text { de café }\end{array}$ & Riesgo de ECV & 9 & $9 / 11$ \\
\hline $\begin{array}{l}\text { Zhang, } \\
\text { et al., } \\
2012\end{array}$ & $\begin{array}{l}\text { Estados } \\
\text { Unidos, } \\
\text { Finlandia, } \\
\text { Suecia, } \\
\text { Holanda, } \\
\text { Italia, } \\
\text { Japón }\end{array}$ & 492,760 & $\begin{array}{l}\text { Población } \\
\text { adulta sana }\end{array}$ & $\begin{array}{l}\text { Consumo } \\
\text { habitual de } \\
\text { café en } \\
\text { diferentes } \\
\text { categorías: } \\
\text { leve, } \\
\text { moderado, } \\
\text { pesado }\end{array}$ & $\begin{array}{l}\text { No } \\
\text { consumo } \\
\text { de café o } \\
\text { consumo } \\
\text { menos de } \\
\text { una vez a la } \\
\text { semana }\end{array}$ & Riesgo de ECV & 13 & $8 / 11$ \\
\hline $\begin{array}{l}\text { Mesas, } \\
\text { et al., } \\
2011\end{array}$ & $\begin{array}{l}\text { Estados } \\
\text { Unidos, } \\
\text { Finlandia, } \\
\text { Suecia }\end{array}$ & $\begin{array}{l}\text { No } \\
\text { especifica }\end{array}$ & $\begin{array}{l}\text { Población } \\
\text { adulta } \\
\text { hipertensa }\end{array}$ & $\begin{array}{l}\text { Consumo de } \\
\text { café o } \\
\text { cafeína en } \\
\text { dosis de } \\
200-300 \text { mg }\end{array}$ & $\begin{array}{l}\text { No } \\
\text { consumo }\end{array}$ & $\begin{array}{l}\text { Riesgo de } \\
\text { muerte por } \\
\text { enfermedad } \\
\text { cardiovascular } \\
\text { y ECV }\end{array}$ & 7 & $9 / 11$ \\
\hline
\end{tabular}

DM2: diabetes mellitus tipo 2; ECV: enfermedad cerebrovascular; IAM: infarto agudo de miocardio. 
Tabla 2. Riesgo de enfermedad cerebrovascular en población general de acuerdo con la categoría de consumo de café

\begin{tabular}{|c|c|c|c|c|c|c|}
\hline \multirow[t]{2}{*}{ Estudio } & $\begin{array}{l}\text { Evento } \\
\text { cerebrovascular }\end{array}$ & $\begin{array}{c}\text { RR para el consumo } \\
\text { de café }\end{array}$ & \multirow{2}{*}{$\begin{array}{l}\text { Número de } \\
\text { estudios } \\
\text { incluidos }\end{array}$} & \multirow{2}{*}{$\begin{array}{l}\text { Calidad de } \\
\text { la revisión } \\
\text { según } \\
\text { AMSTAR }\end{array}$} & \multirow[t]{2}{*}{ Certeza } & \multirow[t]{2}{*}{ Importancia } \\
\hline & $(\%)$ & $($ IC95\%, I) & & & & \\
\hline \multicolumn{7}{|c|}{ Cualquier consumo comparado con no consumo } \\
\hline \multirow{3}{*}{$\begin{array}{l}\text { Zhang, et al., } \\
2012\end{array}$} & \multirow[t]{3}{*}{$12,141 / 492,760(2.46 \%)$} & $\mathrm{RR}=0.89$ & \multirow{3}{*}{$\begin{array}{l}13 \text { cohortes } \\
\text { prospectivas }\end{array}$} & \multirow[t]{3}{*}{$8 / 11$} & \multirow[t]{3}{*}{ Muy baja } & \multirow[t]{3}{*}{ Crítico } \\
\hline & & $(0.81-0.97)$ & & & & \\
\hline & & $(12=69 \%)$ & & & & \\
\hline \multirow{3}{*}{$\begin{array}{l}\text { Kim, et al., } \\
2012\end{array}$} & \multirow[t]{3}{*}{$8110 / 206,437(3.92 \%)$} & $\mathrm{RR}=0.83$ & \multirow{3}{*}{$\begin{array}{l}9 \text { cohortes } \\
\text { prospectivas }\end{array}$} & \multirow[t]{3}{*}{$9 / 11$} & \multirow[t]{3}{*}{ Muy baja } & \multirow[t]{3}{*}{ Crítico } \\
\hline & & $(0.75-0.91)$ & & & & \\
\hline & & $(12=8.2 \%)$ & & & & \\
\hline \multicolumn{7}{|c|}{ Consumo $<3$ tazas al día comparado con no consumo $0<1$ taza a la semana } \\
\hline \multirow{3}{*}{$\begin{array}{l}\text { Larsson y } \\
\text { Orsini, } 2011\end{array}$} & \multirow[t]{3}{*}{$10,003 / 479,689(2.09 \%)$} & $\mathrm{RR}=0.88$ & \multirow{3}{*}{$\begin{array}{l}11 \text { cohortes } \\
\text { prospectivas }\end{array}$} & \multirow[t]{3}{*}{$7 / 11$} & \multirow[t]{3}{*}{ Muy baja } & \multirow[t]{3}{*}{ Crítico } \\
\hline & & $(0.86-0.90)$ & & & & \\
\hline & & SD & & & & \\
\hline \multirow{3}{*}{$\begin{array}{l}\text { Zhang, et al., } \\
2012\end{array}$} & \multirow[t]{3}{*}{$12,141 / 492,760(2.46 \%)$} & $\mathrm{RR}=0.90$ & \multirow{3}{*}{$\begin{array}{l}13 \text { cohortes } \\
\text { prospectivas }\end{array}$} & \multirow[t]{3}{*}{$8 / 11$} & \multirow[t]{3}{*}{ Muy baja } & \multirow[t]{3}{*}{ Crítico } \\
\hline & & $(0.85-0.96)$ & & & & \\
\hline & & $(\mid 2=13 \%)$ & & & & \\
\hline \multicolumn{7}{|c|}{ Consumo de 3 a 4 tazas al día comparado con no consumo } \\
\hline \multirow{3}{*}{$\begin{array}{l}\text { Larsson y } \\
\text { Orsini, } 2011\end{array}$} & \multirow[t]{3}{*}{$10,003 / 479,689(2.09 \%)$} & $\mathrm{RR}=0.88$ & 11 cohortes & $7 / 11$ & Muy baja & Crítico \\
\hline & & $(0.77-1.01)$ & & & & \\
\hline & & SD & & & & \\
\hline Zhang, et al., & $12,141 / 492,760(2,46 \%)$ & $\mathrm{RR}=0.86$ & 13 cohortes & $8 / 11$ & Muy baja & Crítico \\
\hline & & $(0.76-0.97)$ & prospectivas & & & \\
\hline & & $(12=55 \%)$ & & & & \\
\hline
\end{tabular}

Consumo de 40 más tazas al día (hasta 7) comparado con no consumo

\begin{tabular}{|c|c|c|c|c|c|c|}
\hline \multirow{3}{*}{$\begin{array}{l}\text { Larsson y } \\
\text { Orsini, } 2011\end{array}$} & \multirow[t]{3}{*}{$10,003 / 479,689(2.09 \%)$} & $\mathrm{RR}=0.87$ & \multirow{3}{*}{$\begin{array}{l}11 \text { cohortes } \\
\text { prospectivas }\end{array}$} & \multirow[t]{3}{*}{$7 / 11$} & \multirow[t]{3}{*}{ Muy baja } & \multirow[t]{3}{*}{ Crítico } \\
\hline & & $(0.75-1.02)$ & & & & \\
\hline & & SD & & & & \\
\hline \multirow{3}{*}{$\begin{array}{l}\text { Kim, et al., } \\
2012\end{array}$} & \multirow[t]{3}{*}{$8110 / 206,437(3.92 \%)$} & $\mathrm{RR}=0.83$ & \multirow{3}{*}{$\begin{array}{l}9 \text { cohortes } \\
\text { prospectivas }\end{array}$} & \multirow[t]{3}{*}{$9 / 11$} & \multirow[t]{3}{*}{ Muy baja } & \multirow[t]{3}{*}{ Crítico } \\
\hline & & $(0.75-0.91)$ & & & & \\
\hline & & $(12=8.2 \%)$ & & & & \\
\hline \multirow{3}{*}{$\begin{array}{l}\text { Zhang, et al., } \\
2012\end{array}$} & \multirow[t]{3}{*}{$12,141 / 492,760(2.46 \%)$} & $\mathrm{RR}=0.83$ & \multirow{3}{*}{$\begin{array}{l}13 \text { cohortes } \\
\text { prospectivas }\end{array}$} & \multirow[t]{3}{*}{$8 / 11$} & \multirow[t]{3}{*}{ Muy baja } & \multirow[t]{3}{*}{ Crítico } \\
\hline & & $(0.79-0.89)$ & & & & \\
\hline & & $(12=27 \%)$ & & & & \\
\hline \multicolumn{7}{|c|}{ Consumo de más de 7 tazas al día comparado con no consumo } \\
\hline \multirow{3}{*}{$\begin{array}{l}\text { Larsson y } \\
\text { Orsini, } 2011\end{array}$} & \multirow[t]{3}{*}{$10,003 / 479,689(2.09 \%)$} & $\mathrm{RR}=0.93$ & \multirow{3}{*}{$\begin{array}{l}11 \text { cohortes } \\
\text { prospectivas }\end{array}$} & \multirow[t]{3}{*}{$7 / 11$} & \multirow[t]{3}{*}{ Muy baja } & \multirow[t]{3}{*}{ Crítico } \\
\hline & & $(0.76-1.12)$ & & & & \\
\hline & & SD & & & & \\
\hline
\end{tabular}

12: medición estadística de heterogeneidad; RR: riesgo relativo; SD: sin datos. 
para eventos isquémicos con un consumo de 6 o más tazas al día. Dada la heterogeneidad de los estudios, a los autores no les fue posible establecer una medida de asociación ni concluir respecto al papel del café en el riesgo de desarrollar enfermedad cerebrovascular.

\section{Eventos cerebrovasculares isquémicos}

Kim, et al. ${ }^{21}$ encuentran una reducción significativa cuando evalúan el desenlace enfermedad cerebrovascular de subtipo isquémico, comparando diferentes categorías de consumo frente a no consumo, con un $\mathrm{RR}=0.80$ (IC95\%: 0.71-0.90).

De igual forma, en el estudio de Larsson y Orsini ${ }^{22}$ se reporta una reducción significativa de enfermedad cerebrovascular de subtipo isquémico para consumo de café frente a no consumo en los grupos que toman hasta 7 tazas diarias, con reducciones superiores cuanto mayor es el consumo de café ( $R R=0.92, I C 95 \%: 0.87-0.98$ para 1 taza; $\mathrm{RR}=0.87$, IC95\%: $0.79-0.96$ para 2 tazas; $\mathrm{RR}=0.84$, IC95\%: 0.75-0.93 para 3 tazas; $\mathrm{RR}=0.82$ para 4, 5, 6 y 7 tazas, con IC95\% de 0.74-0.91, 0.74$0.90,0.72-0.92$ y $0.70-0.96$, respectivamente).

\section{Eventos cerebrovasculares hemorrágicos}

Ninguno de los estudios evaluados encontró una diferencia significativa en cuanto a reducción de enfermedad cerebrovascular de subtipo hemorrágico, aunque uno halló una tendencia a la reducción ${ }^{22}$.

\section{Diferencias por sexo}

En el estudio de Kim, et al. ${ }^{21}$ se encontró una reducción estadísticamente significativa para las mujeres en el grupo de ingesta alta (4 o más tazas de café), con un $\mathrm{RR}=0.81$ (IC95\%: 0.70 a 0.93), dato que también se encontró en el metaanálisis con mayor número de eventos reportados, con una reducción del riesgo de enfermedad cerebrovascular del 17\% (IC95\%: 0.790.87 ) en el grupo de mujeres. Aunque este mismo estudio no encontró resultados significativos en los hombres, sí halló una tendencia a la reducción del riesgo en este grupo ${ }^{20}$.

Larsson y Orsini ${ }^{22}$ reportan que un consumo de hasta 6 tazas, comparado con el no consumo, se asocia con reducciones significativas del riesgo de enfermedad cerebrovascular en los hombres entre el $10 \%$ y el $20 \%$, aunque no lineales; los consumos superiores no muestran asociación significativa. Para el caso de las mujeres, el beneficio se encontró con 1 a 2 tazas al día (RR $=0.87$,
IC95\%: 0.78-0.97 para 1 taza, y RR = 0.84, IC95\%: 0.740.95 para 2 tazas), con una tendencia a la reducción, pero con resultados no significativos para consumos de 3,4 y 5 tazas, y sin que fuera posible evaluar las categorías de consumo mayor por ausencia de datos ${ }^{22}$.

\section{Mortalidad por enfermedad cerebrovascular}

Se incluyeron tres metaanálisis que evaluaron el desenlace de mortalidad asociada específicamente a enfermedad cerebrovascular. El estudio de Grosso, et al. ${ }^{24}$ encontró reducciones significativas en cuanto a mortalidad por enfermedad cerebrovascular, con $\mathrm{RR}=0.72$ (IC95\%: 0.6-0.87) al comparar el no consumo con el consumo de 4 tazas diarias. Por otra parte, comparando el no consumo contra cualquier categoría desde 1 hasta 6 tazas, se mantiene el efecto positivo (Fig. 1). Para consumos superiores, el resultado deja de ser significativo, pero se mantiene la tendencia.

El análisis por subgrupos evidencia que en el grupo de fumadores la reducción del riesgo se mantiene y es significativa para las categorías de consumo de 2 a 7 tazas, comparado con consumos inferiores. En el subgrupo de no fumadores se mantiene la tendencia, pero el resultado no es estadísticamente significativo (Fig. 2). A diferencia de lo anterior, los estudios de Malerba, et al. ${ }^{25} \mathrm{y}$ de Mesas et al. ${ }^{23}$ no encuentran diferencias significativas entre consumo de café y mortalidad por enfermedad cerebrovascular.

\section{Discusión}

El consumo de café se asocia con cambios en la circulación cerebral y reducción del flujo en este órgano; además, según algunos autores, el consumo moderado de esta sustancia puede producir incrementos transitorios de las cifras de presión arterial. Sin embargo, otros componentes del café, diferentes de la cafeína, pueden tener efectos protectores y antioxidantes que mejoran la función endotelial, disminuyen la respuesta inflamatoria e incrementan la sensibilidad a la insulina, entre otros efectos con posible beneficio cardiovascular ${ }^{21,22}$.

En esta revisión se encontró que el consumo de café a diferentes dosis se asocia con reducciones globales en el riesgo de eventos cerebrovasculares. La evidencia resultó ser significativa en dos de los tres metaanálisis que incluyen este desenlace. Destaca que las dos primeras referencias, los estudios de Zhang, et al. ${ }^{20} \mathrm{y}$ de Kim, et al. ${ }^{21}$, se caracterizan por abarcar múltiples regiones y tener suficientes tamaños de muestra y de 


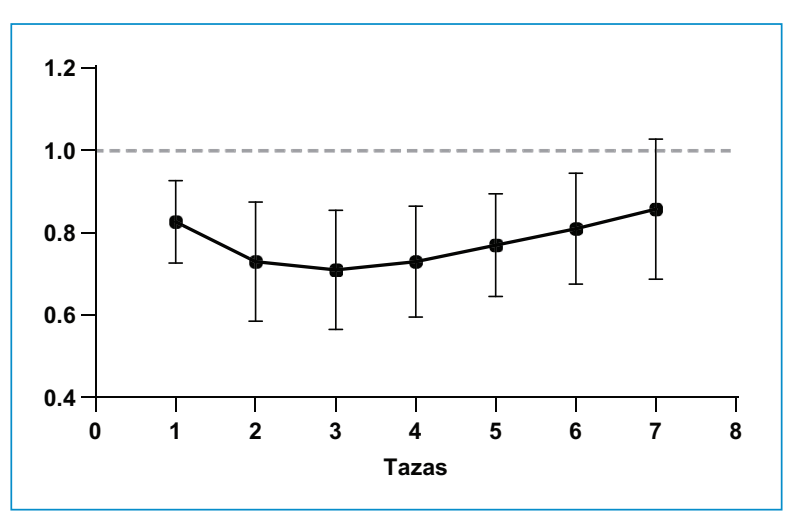

Figura 1. Riesgo de muerte por enfermedad cerebrovascular de acuerdo con el número de tazas de café consumidas en población general.

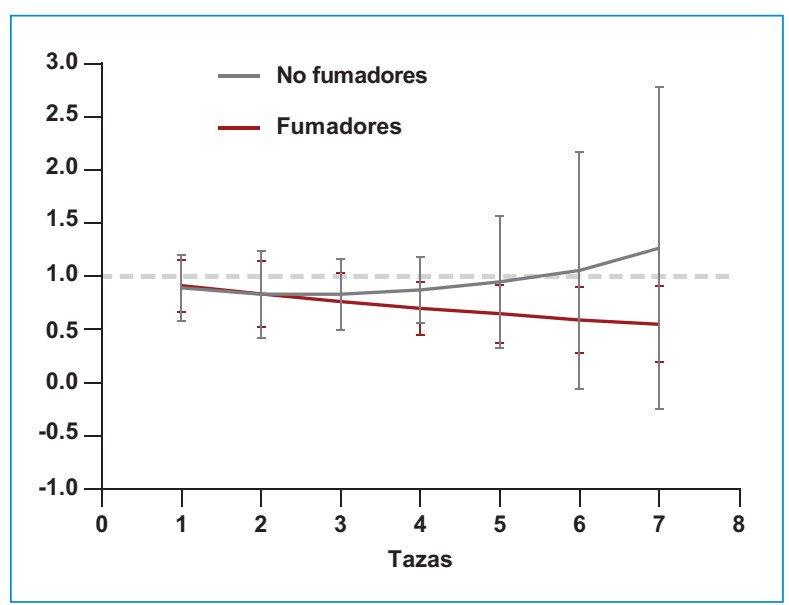

Figura 2. Riesgo de muerte por enfermedad cerebrovascular de acuerdo con ser o no ser fumador y el número de tazas de café consumidas.

eventos, lo cual permite inferir la posibilidad de que el efecto sea independiente de la raza, y aunque el tercer estudio no reporta un desenlace estadísticamente significativo, cabe anotar que sí demuestra una tendencia a la reducción del riesgo. Los hallazgos sugieren que la reducción del riesgo de enfermedad cerebrovascular podría estar asociada con la cantidad de consumo, de modo que habría mayor protección con el consumo de rango moderado (hasta 4 tazas diarias de café).

Con relación a las diferencias por sexo, en la mayoría de los estudios se encontró que la protección conferida por el café es mayor en las mujeres, en rangos de consumo moderado. Esto concuerda con publicaciones recientes que han descrito una asociación inversa entre el consumo de café y el riesgo de mortalidad por enfermedad cerebrovascular, además de evidencia bioquímica de perfiles metabólicos menos aterogénicos, particularmente en el sexo femenino ${ }^{11}$. Sin embargo, cuando se evalúa la mortalidad por enfermedad cerebrovascular en términos globales, la evidencia es conflictiva: algunos estudios demuestran reducciones significativas y lineales respecto al consumo de café, en tanto que otros no logran demostrar el efecto protector de la bebida ${ }^{23-25}$.

La evidencia actual no permite distinguir si el efecto protector que confiere el café se puede extrapolar a todos los subtipos de enfermedad cerebrovascular; el efecto parece ser claro para los eventos isquémicos, pero se requieren estudios que aborden este tema.

De acuerdo con la evidencia analizada, el café, en consumos moderados a altos, y en particular para el sexo femenino, confiere un efecto protector en relación con la aparición de eventos cerebrovasculares de tipo isquémico, hallazgo que concuerda en los metaanálisis incluidos en esta revisión.

Estos hallazgos concuerdan con los de otras publicaciones que han encontrado asociaciones favorables entre el consumo de café y la reducción de eventos cerebrovasculares, en particular para el sexo femenino $0^{11,26}$.

Cabe señalar que las publicaciones elegidas no presentan sesgos de publicación y que dos de los metaanálisis no tienen sesgo geográfico por la inclusión de varias regiones del mundo.

Esta revisión encontró varias limitaciones. Por una parte, no hay estandarización en las variables, como el tipo de preparación (filtrado, expreso, hervido, etc.), el tipo de café (liofilizado, en grano, molido, cafeinado, etc.), el tamaño de la taza y, sobre todo, la cantidad real ingerida. Por otra parte, el tipo de intervención hace difícil cuantificar objetivamente el consumo, ya que los datos suelen obtenerse mediante reporte de los sujetos de estudio, lo cual plantea la posibilidad de un sesgo por autorreporte. Finalmente, a excepción de los estudios de Zhang, et al. ${ }^{20}$ y de Grosso, et al. ${ }^{24}$, los demás estudios no controlaron desenlaces por variables de confusión, como tabaquismo, hipertensión arterial o consumo de alcohol, lo cual limita la interpretación de los resultados.

\section{Conclusiones}

Con un grado de certeza muy bajo y un nivel de importancia crítico según la clasificación GRADE, los estudios demuestran una asociación favorable entre el consumo de café y la reducción del riesgo de enfermedad cerebrovascular, la cual es estadísticamente 
significativa para las mujeres. Sería interesante evaluar la evidencia de acuerdo con subtipos de enfermedad cerebrovascular, tipo de café y tipo de preparación, entre otros. Para consolidar la evidencia es importante estandarizar las categorías de consumo. No obstante, estos resultados son relevantes puesto que permiten eliminar mitos y barreras respecto al consumo de café para poblaciones en riesgo o con antecedente de enfermedad cerebrovascular, lo cual, para un país como Colombia, resulta importante en lo que atañe a implicaciones culturales, sociales y agroeconómicas.

\section{Respuesta a la paciente}

Hay evidencia científica que asocia el consumo de café con un menor riesgo de padecer eventos cerebrovasculares, tanto isquémicos como hemorrágicos, aunque se requieren estudios complementarios. Lo que sí está claro es que no hay evidencia de que el consumo de café incremente el riesgo de enfermedad cerebrovascular.

Teniendo en cuenta lo anterior, a la paciente se le recomendaría continuar consumiendo café como lo ha hecho tradicionalmente. Por otra parte, deben favorecerse los estilos de vida saludables y el control de la presión arterial, ya que la evidencia es innegable respecto al beneficio de estas intervenciones para los desenlaces en salud.

\section{Financiamiento}

El trabajo que permitió la elaboración de este artículo no contó con financiación pública ni privada de terceros.

\section{Conflicto de intereses}

Los autores declaran no tener ningún tipo de conflicto de intereses.

\section{Responsabilidades éticas}

Protección de personas y animales. Los autores declaran que para esta investigación no se han realizado experimentos en seres humanos ni en animales.

Confidencialidad de los datos. Los autores declaran que en este artículo no aparecen datos de pacientes.

Derecho a la privacidad y consentimiento informado. Los autores declaran que en este artículo no aparecen datos de pacientes.

\section{Bibliografía}

1. Sacco RL, Kasner SE, Broderick JP, Caplan LR, Connors JJ, Culebras A, et al. An updated definition of stroke for the $21^{\text {st }}$. century: a statement for healthcare professionals from the American Heart Association/American Stroke Association. Stroke. 2013:44:2064-89.

2. Instituto Nacional de Salud, Observatorio Nacional de Salud. Quinto informe ONS. Carga de enfermedad por enfermedades crónicas no transmisibles y discapacidad en Colombia. Vol. V. Edición 2015. [Consultado el 10 de mayo de 2018]. Disponible en: https://www.minsalud.gov.co/sites/rid/Lists/BibliotecaDigital/RIDE/IA/INS/informe-ons-5.pdf.

3. Silva FA, Zarruk JG, Quintero C, Arenas W, Silva SY. Enfermedad cerebrovascular en Colombia. Rev Colomb Cardiol. 2006;13:85-9.

4. SASI Group (University of Sheffield), Newman M (University of Michigan). Coffee consumption. 2006. Disponible en: http://archive.worldmapper. org/posters/worldmapper 1038 coffee consumption ver2.pdf.

5. Neumann DM (Neumann Kaffee Gruppe). The World of Coffee in 2017 San José de Costa Rica; 2012. Disponible en: https://www.sintercafe. com/uploads/File/2012/presentations/0.neumann.pdf.

6. Federación Nacional de Cafeteros. Café y salud. Verdades y mitos del café. [Consultado el 1 de julio de 2018]. Disponible en: https://www.federaciondecafeteros.org/static/pergamino-fnc/uploads/cafeSalud.pdf.

7. Echeverri D, Buitrago L, Montes F, Mejía I, González MP. Café para cardiólogos. Rev Colomb Cardiol. 2005;11:357-65.

8. Diez mitos y verdades del café. El Espectador, 27 de junio de 2016. [Consultado el 1 de julio de 2018]. Disponible en: https://www.elespectador.com/cromos/gastronomia/diez-mitos-y-verdades-del-cafe-20088.

9. Park S-Y, Freedman ND, Haiman CA, Le Marchand L, Wilkens LR, Setiawan VW. Association of coffee consumption with total and cause-specific mortality among nonwhite populations. Ann Intern Med. 2017;167:228.

10. Ding M, Bhupathiraju SN, Satija A, Van Dam RM, Hu FB. Long-term coffee consumption and risk of cardiovascular disease: a systematic review and a dose-response meta-analysis of prospective cohort studies. Circulation. 2014;129:643-59.

11. Gunter MJ, Murphy N, Cross AJ, Dossus L, Dartois L, Fagherazzi G, et al. Coffee drinking and mortality in 10 European countries: a multinational cohort study. Ann Intern Med. 2017;167:236-47.

12. Lamuela-Raventos RM, Quifer-Rada P. Effect of dietary polyphenols on cardiovascular risk. Heart. 2016;102:1340-1.

13. Larsson SC. Coffee, tea, and cocoa and risk of stroke. Stroke. 2014;45:309-14.

14. Otto CM. Heartbeat: dietary polyphenols and vascular function. Heart. 2016;102:1337-8.

15. Watanabe T, Arai $Y$, Mitsui $Y$, Kusaura T, Okawa W, Kajihara Y, et al. The blood pressure-lowering effect and safety of chlorogenic acid from green coffee bean extract in essential hypertension. Clin Exp Hypertens. 2006;28:439-49

16. Zhao $\mathrm{Y}$, Wang J, Ballevre $\mathrm{O}$, Luo $\mathrm{H}$, Zhang W. Antihypertensive effects and mechanisms of chlorogenic acids. Hypertens Res. 2012;35:370-4.

17. Alba Talero LH, Peñaloza MJ, Gutiérrez V, Castillo JS. Efecto del consumo habitual de café en la salud cardiovascular: protocolo de una revisión de revisiones sistemáticas de la literatura. Univ Médicas. 2019;60(2).

18. GRADEpro. GDT: GRADEpro Guideline Development Tool. McMaster University, Evidence Prime Inc., Ontario (Canada); 2015. Disponible en: https://gradepro.org/

19. GraphPad Software Inc. GraphPad Prism Version 8.0.1 for Windows. San Diego, California, USA. Disponible en: www.graphpad.com.

20. Zhang R, Wang Y, Song B, Jørgensen HS, Xu Y. Coffee consumption and risk of stroke: a meta-analysis of cohort studies. Cent Eur J Med. 2012;7:310-6.

21. Kim B, Nam Y, Kim J, Choi H, Won C. Coffee consumption and stroke risk: a meta-analysis of epidemiologic studies. Korean $\mathrm{J}$ Fam Med. 2012;33:356-65.

22. Larsson SC, Orsini N. Coffee consumption and risk of stroke: a dose-response meta-analysis of prospective studies. Am J Epidemiol. 2011;174:993-1001.

23. Mesas AE, Rodríguez-Artalejo F, León-Muñoz LM, López-García E, Nurminen ML, Niittynen L, et al. The effect of coffee on blood pressure and cardiovascular disease in hypertensive individuals: a systematic review and meta-analysis. Am J Clin Nutr. 2011;94:1113-26.

24. Grosso G, Micek A, Godos J, Sciacca S, Pajak A, Martínez-González MA et al. Coffee consumption and risk of all-cause, cardiovascular, and cancer mortality in smokers and non-smokers: a dose-response meta-analysis. Eur J Epidemiol. 2016;31:1191-205.

25. Malerba S, Turati F, Galeone C, Pelucchi C, Verga F, La Vecchia C, et al. A meta-analysis of prospective studies of coffee consumption and mortality for all causes, cancers and cardiovascular diseases. Eur $\mathrm{J}$ Epidemiol. 2013:28:527-39.

26. Lee JJE, Kim Y. Relationship between coffee consumption and stroke risk in Korean population: the Health Examinees (HEXA) Study. Nutr J. 2017;16:1-8. 